\title{
Microestructuras producidas en la soldadura de unión de aceros para tuberías de gran diámetro $^{(\bullet)}$
}

\author{
M. Zalazar ${ }^{(*)}$, H.J. Quesada ${ }^{(*)}$ y E.P. Asta ${ }^{(* *)}$ \\ Resumen En este trabajo se estudian las microestructuras producidas en la zona afectada por el calor (HAZ) de \\ la soldadura de unión en aceros utilizados en la construcción de poliductos. Se analizan las juntas \\ obtenidas mediante soldadura manual con electrodo revestido. A pesar de que este es un proceso de \\ soldadura con un bajo aporte térmico, se encuentran zonas frágiles locales que se originan por un \\ mecanismo complejo influido por parámetros de soldadura y composición química del acero. Se ana- \\ lizan secuencias de soldaduras que ayuden a la desaparición de las mismas.
}

Palabras clave: Aceros HSLA. Soldadura manual con electrodo revestido. Zona afectada por el calor. Microestructura. Ensayo Charpy V.

\section{Microstructure produced in the welding of steels for wide diameter pipes}

\begin{abstract}
This is an evaluation of the microstructure in the heat affected zone (HAZ) of steel weldings used in pipe lines. The welding was made by shielding metal arc welding. This is a welding procces with multiple run and low heat input, however local brittle zones are present in the HAZ. These are originated by a complex mechanism that is influenced by welding parameters and chemical composition of the steel. The welding sequences that help to eliminate the local brittle zones are studied.
\end{abstract}

Keywords: HSLA steels. Shielding metal arc welding. Heat affected zone. Microstructure.

\section{INTRODUCCIÓN}

La construcción de poliductos para largos trayectos conlleva la utilización de aceros de alta resistencia y baja aleación (HSLA) en los cuales una combinación de composición química y laminación termomecánica permite incrementar la tensión de fluencia y mejorar la tenacidad junto con la soldabilidad, lo que se logra a través de una disminución del car-

$(\bullet \quad$ Trabajo recibido el día 19 de enero de 1998 y aceptado en su forma final el 12 de septiembre de 1998.

(*) Laboratorio de Metalografía. Dpto. de Mecánica Aplicada. Facultad de Ingeniería. Universidad Nacional del Comahue. Buenos Aires 1400. 8300-Neuquen (Argentina).

(**) Universidad Tecnológica Nacional. Regional Haedo. Paris 532. 1706-Haedo. Buenos Aires (Argentina). bono equivalente que permite la eliminación de tratamientos térmicos de pre y post-soldadura.

En la unión de estos materiales aunque se ha incrementado el uso de procesos semiautomáticos, la soldadura manual con electrodo revestido continúa imponiéndose mayoritariamente. Si bien existen normas que permiten evaluar las propiedades mecánicas de la unión, suelen aparecer problemas de tenacidad asociados con la zona afectada por el calor (HAZ), cuyo origen no se encuentra adecuadamente clarificado.

En el proceso de soldadura manual de poliductos se requiere un diseño de junta en $\mathrm{V}$ y varias pasadas de soldaduras, las que generan en el metal base una HAZ muy compleja, con propiedades mecánicas no siempre adecuadas, debido a que la sucesión de ciclos térmicos produce problemas tales como crecimiento de grano, formación de fases frágiles cercanas a la línea de fusión, inclusiones no metálicas y ablandamiento excesivo ( 1 y 2 ). 
La figura 1 (3) muestra esquemáticamente los cambios de microestructura que se producen en una soldadura de pasadas múltiples. La zona afectada por el calor, cuya microestructura es engrosada por el primer ciclo, cambia a diferentes microestructuras de acuerdo a la secuencia térmica de las pasadas subsiguientes. Cuando la temperatura máxima de la segunda pasada está inmediatamente por encima de la línea crítica superior $\left(A_{C 3}\right)$, la microestructura se refina, lo que suele llamarse efecto de normalizado (C en la Fig. 1); cuando la temperatura es menor que $\mathrm{A}_{\mathrm{Cl}}$, la microestructura es solamente revenida (A en la Fig. 1). En el caso de que la temperatura de recalentamiento se encuentre entre $A_{C 3}$ y $A_{C 1}$ (denominada zona intercrítica de temperaturas) se generan regiones locales enriquecidas en carbono con riesgo de formarse microestructuras frágiles (B en la Fig. 1). Esta región de fase dual también existe aproximadamente a $3 \mathrm{~mm}$ de la línea de fusión de la primera pasada.

A través de la simulación de ciclos térmicos de soldadura se determinó que, desde el punto de vista de microestructuras, el mayor deterioro en la tenacidad de la HAZ se debe a la formación de zonas frágiles locales (LBZ) (otros factores son crecimiento de grano y presencia de impurezas), los autores (4 y 5 ) coinciden en establecer que las mis- mas se originan en una soldadura de pasadas múltiples, principalmente en la región identificada como zona de grano grueso recalentada en la región intercrítica de temperaturas. Las LBZ son fuertemente afectadas por factores metalúrgicos tales como las islas de martensita, por lo que su presencia se ve influida por la composición química de la aleación y por la sucesión de ciclos térmicos a la que se somete el metal base.

Algunos autores (6) reportan ecuaciones que vinculan la influencia de las LBZ sobre la temperatura de transición; otros, en cambio, consideran que el deterioro de las propiedades mecánicas debido a las LBZ no depende del porcentaje de las mismas. También debería considerarse en el análisis el aspecto morfológico de las LBZ que pueden presentar una forma alargada, cuando la relación longitud/anchura de la fase es $4 / 1$. En este punto existe discrepancia en cuanto a su influencia sobre las propiedades mecánicas. En los aceros con níquel, molibdeno y mayores porcentajes de carbono, se forman ambos tipos de LBZ, y junto con la matriz deterioran la tenacidad del metal base. El tiempo de enfriamiento, del segundo ciclo de soldadura, entre 800 y $500{ }^{\circ} \mathrm{C}\left(\Delta t_{8 / 5}\right)$ influye no sólo en la aparición de estas fases, sino también en la morfología que presentan.

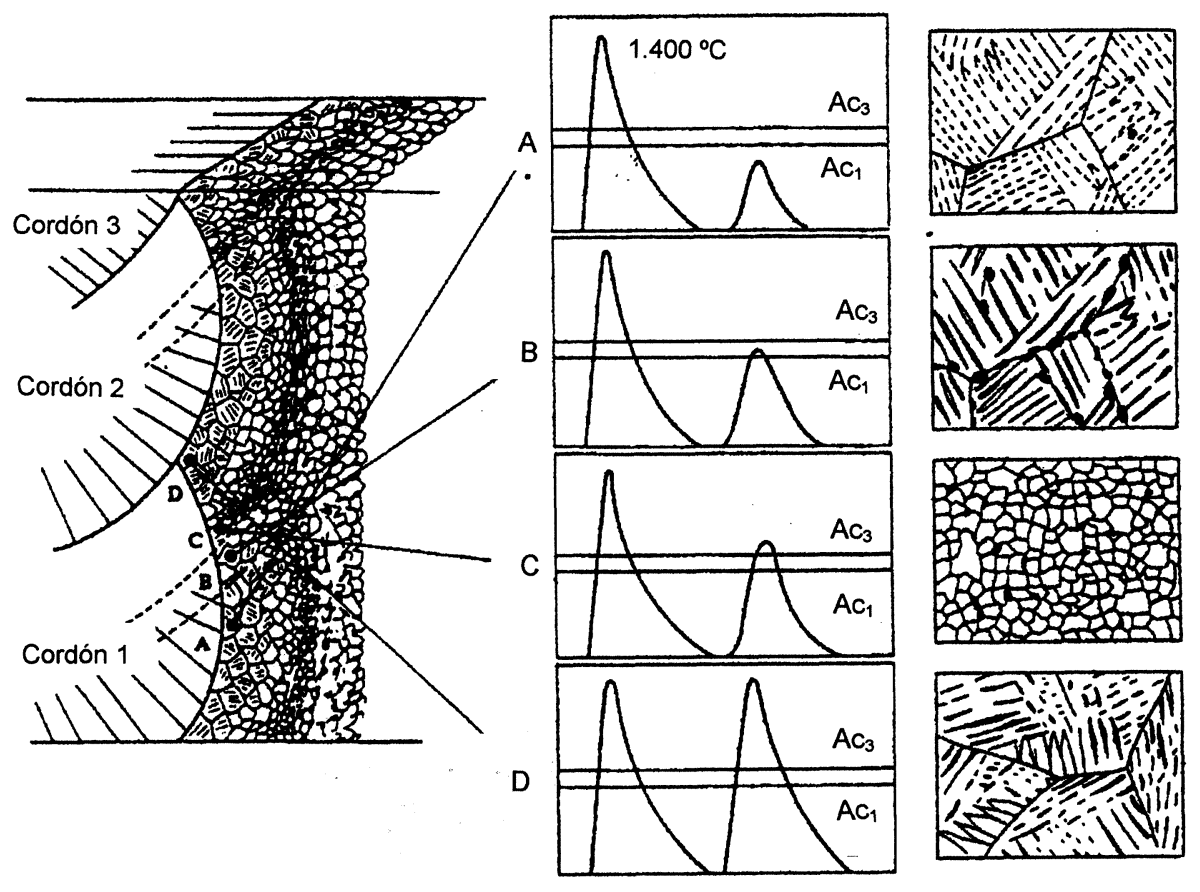

FIG. 1.- Esquema de la relación entre microestructura y ciclo térmico en la HAZ (3).

FIG. 1.- Schematic diagram showing the relation between microstructure and thermal cycle in the HAZ (3). 
Desde el punto de vista de la composición química, las LBZ se forman más fácilmente por la adición de boro, nitrógeno y carbono, así como elementos formadores de carburos (molibdeno, niobio y vanadio); estos últimos retardan la difusión de carbono durante la transformación desde la austenita a ferrita y la descomposición de las LBZ durante el revenido. Para algunos autores, el efecto del manganeso es menos efectivo. Por otro lado, la reducción de silicio ( 7 y 8) suprime la formación de las islas y actúa favoreciendo la disolución de las mismas durante el revenido a temperaturas inferiores, efecto que es difícil de cuantificar con las ecuaciones de soldabilidad.

\section{MATERIAL Y MÉTODO}

Se realizaron uniones en aceros API 5L X60 en tuberías de distintos diámetros y espesores de pared, mediante el proceso de soldadura manual con electrodo revestido. La tabla 1 indica las composiciones químicas de los aceros empleados, y la tabla 11 , los parámetros utilizados en la realización de las uniones; en todos los casos se empleó un diseño de junta establecido por las Normas. Los procedimientos 5 y 6 se efectuaron sobre el mismo acero tomando como base los resultados previos que llevaron a recomendar un control de la temperatura entre pasadas.

Se prepararon probetas tomándolas en sentido transversal a la unión. Sobre ellas se realizaron barridos de microdureza y ensayos de Charpy $\mathrm{V}$ ubicando la entalla sobre la HAZ, para lo cual se realizaron previamente macrografias de la región. La figura 2 indica el sentido de extracción de las probetas. Posterior al ensayo Charpy V, se observó la superficie de fractura y se analizó la microestructura que dio origen a la misma.

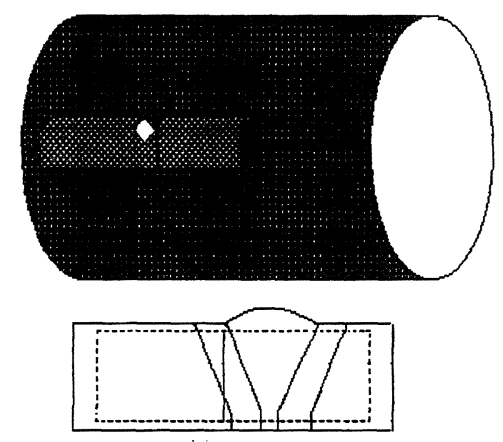

a)

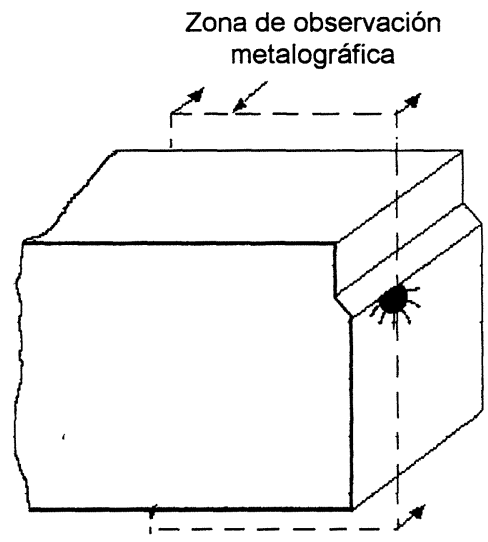

b)

FIG. 2.- Probetas para: (a) Charpy V. (b) y metalografía.

FIG. 2.- Specimens from: (a) Charpy V. (b) metallography.

\subsection{Temperatura de precalentamiento}

Existen numerosos métodos propuestos para determinar o estimar la necesidad de precalentar en la soldadura de aceros. Estos métodos consideran algunos o todos los factores que influyen en la fisuración en frío, como son: composición química del acero, difusión de hidrógeno, calor aportado,

TABLA I.- Composición química

TABLE I.- Chemical composition

\begin{tabular}{|c|c|c|c|c|c|c|c|c|c|c|c|c|c|c|c|c|c|c|c|}
\hline Material & \multicolumn{11}{|c|}{ Composición química, \% } & \multicolumn{10}{c|}{$\mathrm{C}_{\text {eq }}, \%$} \\
\hline API 5LX60 & $\mathrm{C}$ & $\mathrm{Mn}$ & $\mathrm{P}$ & $\mathrm{S}$ & $\mathrm{Si}$ & $\mathrm{Cr}$ & $\mathrm{Cu}$ & $\mathrm{Mo}$ & $\mathrm{Ni}$ & $\mathrm{Al}$ & $\mathrm{Co}$ & $\mathrm{V}$ & $\mathrm{Ti}$ & $\mathrm{Sn}$ & $\mathrm{As}$ & $\mathrm{W}$ & $\mathrm{Nb}$ & $\mathrm{Pcm}$ & $\mathrm{CEIIW}$ \\
\hline Tubo núm. 2 & 0,12 & 1,3 & 0,013 & 0,005 & 0,24 & 0,02 & 0,01 & 0,01 & 0,03 & 0,05 & 0,01 & 0,05 & 0,01 & 0,01 & 0,01 & 0,01 & 0,02 & 0,201 & 0,355 \\
Tubo núm. 3 & 0,11 & 1,28 & 0,011 & 0,004 & 0,23 & 0,02 & 0,01 & 0,01 & 0,03 & 0,05 & 0,01 & 0,04 & 0,01 & 0,01 & 0,01 & 0,01 & 0,02 & 0,188 & 0,34 \\
Tubo núm. 4 & 0,12 & 1,24 & 0,012 & 0,004 & 0,26 & 0,02 & 0,01 & 0,01 & 0,03 & 0,05 & 0,01 & 0,04 & 0,01 & 0,01 & 0,01 & 0,01 & 0,02 & 0,197 & 0,343 \\
Tubo núm. 5/6 & 0,1 & 1,42 & 0,023 & 0,012 & 0,17 & 0,023 & 0,006 & 0 & 0,023 & 0,026 & 0 & 0,028 & 0,013 & 0 & 0 & 0 & 0 & 0,181 & 0,349 \\
\hline
\end{tabular}

$\mathrm{Pcm}=\mathrm{C}+\mathrm{Si} / 30+(\mathrm{Mn}+\mathrm{Cu}+\mathrm{Cr}) / 20+\mathrm{Ni} / 60+\mathrm{Mo} / 15+\mathrm{V} / 10+5 * \mathrm{~B}$

$\mathrm{CEIIW}=\mathrm{C}+\mathrm{Mn} / 6+(\mathrm{Cr}+\mathrm{Mo}+\mathrm{V}) / 5+(\mathrm{Cu}+\mathrm{Ni}) / 15$. 
TABLA II.- Parámetros y diseños de juntas

TABLE II.- Parameters and design of joints

\begin{tabular}{|c|c|c|c|c|c|c|c|c|c|c|c|c|}
\hline \multirow[b]{2}{*}{ Pasadas } & \multicolumn{6}{|c|}{ Tubo núm. 2} & \multicolumn{6}{|c|}{ Tubo núm. 3} \\
\hline & 1 & 2 & 3 & 4 & 5 & 6 & 1 & 2 & 3 & 4 & 5 & 6 \\
\hline Intensidad, A & 162 & 183 & 191 & 190 & 165 & 158 & 152 & 158 & 140 & 110 & 105 & 120 \\
\hline Tensión, V & 25 & 32 & 35 & 33 & 30 & 31 & 34 & 35 & 28 & 28 & 28 & 29 \\
\hline Velocidad, $\mathrm{mm} / \mathrm{s}$ & 7,17 & 5 & 4,67 & 5,33 & 4,67 & 4,17 & 4,95 & 5,86 & 3,43 & 2,6 & 2,94 & 3,62 \\
\hline Energía, kJ/mm & 0,57 & 1,17 & 1,43 & 1,18 & 1,06 & 1,18 & 1,04 & 0,94 & 1,14 & 1,18 & 1,00 & 0,96 \\
\hline Polaridad CC $-/+$ & $(+)$ & $(+)$ & $(+)$ & $(+)$ & $(+)$ & $(+)$ & $(-)$ & $(+)$ & $(+)$ & $(+)$ & $(+)$ & $(+)$ \\
\hline Temp. M.B., ${ }^{\circ} \mathrm{C}$ & 43 & 70 & 88 & 110 & 120 & 117 & 42 & 73 & 68 & 100 & 85 & 90 \\
\hline Diámetro, mm & 4 & 5 & 5 & 5 & 5 & 5 & 4 & 4 & 4 & 4 & 4 & 4 \\
\hline Electrodo - AWS & $*$ & $* *$ & $* *$ & $* *$ & $* *$ & $* *$ & $*$ & $* * *$ & $* * *$ & $* * *$ & $* * *$ & $* * *$ \\
\hline e & $-s \leftleftarrows 1$ & & & & $\begin{array}{l}e=10,3 \\
\beta=30^{\circ} \\
t=1,6 n \\
s=1,6 n\end{array}$ & & & & $\begin{array}{l}e=10,3 \\
\beta=37^{\circ} \\
t=2 \mathrm{mn} \\
\mathrm{s}=2 \mathrm{mr}\end{array}$ & & & \\
\hline
\end{tabular}

\begin{tabular}{|c|c|c|c|c|c|c|c|c|c|c|c|c|c|c|}
\hline & \multicolumn{5}{|c|}{ Tubo núm. 4} & \multicolumn{5}{|c|}{ Túbo núm. 5} & \multicolumn{4}{|c|}{ Tubo núm. 6} \\
\hline & 1 & 2 & 3 & 4 & 5 & 1 & 2 & 3 & 4 & 5 & 1 & 2 & 3 & 4 \\
\hline Intensidad, A & 130 & 153 & 152 & 150 & 158 & 145 & 195 & 170 & 160 & 122 & 180 & 170 & 140 & 117 \\
\hline Tensión, V & 38 & 27 & 27 & 27 & 27 & 26 & 32 & 31 & 30 & 27 & 32 & 31 & 30 & 28 \\
\hline Velocidad, $\mathrm{mm} / \mathrm{s}$ & 4,28 & 4,09 & 3,6 & 3,45 & 3,57 & 6,20 & 9,7 & 6,5 & 5,4 & 3,3 & 7,10 & 8,6 & 6,5 & 5 \\
\hline Energía. $\mathrm{kJ} / \mathrm{mm}$ & 1,15 & 1,01 & 1,14 & 1,17 & 1,19 & 0,61 & 0,64 & 0,8 & 0,89 & 1,00 & 0,81 & 0,61 & 0,65 & 0,66 \\
\hline Polaridad CC $-1+$ & $(-)$ & $(+)$ & $(+)$ & $(+)$ & $(+)$ & $(+)$ & $(+)$ & $(+)$ & $(+)$ & $(+)$ & $(+)$ & $(+)$ & $(+)$ & $(+)$ \\
\hline Temp. M.B., ${ }^{\circ} \mathrm{C}$ & 30 & 52 & 50 & 70 & 82 & 30 & 48 & 55 & 60 & 60 & 80 & 80 & 80 & 80 \\
\hline Diámetro, mm & 3 & 4 & 4 & 4 & 4 & 4 & 4 & 4 & 4 & 4 & 4 & 4 & 4 & 4 \\
\hline Electrodo - AWS & * & **** & **** & **** & *** & $* * * *$ & $* * * *$ & ****** & $* * * *$ & ***** & ***** & ****** & $* * * *$ & ***** \\
\hline e & $s_{+}$ & & & & $\begin{array}{l}e=10 \\
\beta=3 \\
t=2 \\
s=2\end{array}$ & $\begin{array}{l}, 3 \mathrm{~mm} \\
0^{\circ} \\
\mathrm{nm} \\
\mathrm{mm}\end{array}$ & & & $\begin{array}{l}e=6,4 \mathrm{~m} \\
\beta=30^{\circ} \\
t=2,2 \mathrm{mr} \\
\mathrm{s}=1,6 \mathrm{mI}\end{array}$ & & & $\begin{array}{l}\mathrm{t}=6, \\
\beta=3 \\
\mathrm{t}=2, \\
\mathrm{~s}=2 \mathrm{i}\end{array}$ & $\begin{array}{l}4 \mathrm{~mm} \\
2^{\circ} \\
5 \mathrm{~mm} \\
\mathrm{~mm}\end{array}$ & \\
\hline
\end{tabular}

*E610 ***E7010-G

$* * \mathrm{E} 7010 \mathrm{~A} 1 \quad * * * * \mathrm{E} 8010-\mathrm{G}$

espesor del metal base, tensiones residuales en la soldadura y embridado de la junta. Sin embargo, hay una considerable diferencia en la valoración de la importancia de estos factores entre los distintos métodos. Por ejemplo, el efecto de la composición química difiere de un método a otro en la evaluación de la importancia de cada elemento de aleación, y, por lo tanto, se obtienen distintos valores de carbono equivalente.

Se analizaron los métodos propuestos por Coe (9); por la British Standard BS 5135 (10); por el Instituto Internacional de la Soldadura (IIW) (9); por el método denominado "control de la temperatura (9); por el método de la carta (11); por el método que recomienda la Norma ANSI/AWS D 1.1-94
(12) y por el que recomienda Suzuki H (13). Con todos ellos se determinó que no se requería precalentar, ya que la temperatura de los tubos antes de soldarlos era superior a los $30^{\circ} \mathrm{C}$. Sin embargo, también se realizaron uniones soldadas controlando la temperatura de precalentamiento y entre pasadas en $80^{\circ} \mathrm{C}$ aproximadamente (tubo núm. 6).

\subsection{Estudios macro y microscópicos}

De todas las muestras se obtuvieron probetas en sentido transversal a la unión. En un principio se observan las uniones globalmente para luego analizar las zonas parciales. 
Se utilizan dos técnicas de ataque para definir adecuadamente las fases de interés. Un ataque con Nital $2 \%$, seguido del reactivo Le Pera (14) y un doble ataque electrolítico (15). Ambos reactivos dan una adecuada definición de las zonas locales frágiles en el microscopio óptico, lo que permite su cuantificación. En los casos donde la soldadura total presenta microestructuras susceptibles, se analizan pasadas previas para determinar el efecto del revenido de las mismas.

\section{RESULTADOS Y DISCUSIÓN}

El metal base presenta una microestructura de ferrita y perlita en forma de bandas; esto, como es de esperar, genera heterogeneidades químicas en la HAZ. Todas las muestras analizadas presentan LBZ sobre esta zona. La figura 3 muestra el aspecto de estas fases por microscopia óptica y electrónica. La figura 3a se obtuvo utilizando el ataque Nital más Le Pera. La figura $3 b$ se obtuvo utilizando un doble ataque electrolítico.

Se observa que las fases se originan en la tercera pasada de soldadura (posteriormente a la denominada pasada en caliente), no siendo posible su eliminación, siendo, por otro lado, general sobre toda la HAZ, no pudiéndose distinguir una ubicación preferencial en la zona de grano grueso recalentada en el rango intercrítico de temperatura lo que, probablemente, se deba a que la distancia entre las isotermas es poca, con lo cual se observa un constante cambio en las microestructuras. Las probetas 2, 3, 4 y 5 presentan fases en formas alargadas y masivas, mientras que la muestra 6 , que tiene una temperatura entre pasadas de $80{ }^{\circ} \mathrm{C}$, presenta fases en forma masivas con un menor tamaño.

La tabla 111 muestra los resultados obtenidos en los ensayos Charpy V. Se debe destacar el uso de distinto tamaño de probetas así como las temperaturas de ensayo. Posteriormente al ensayo, se observan las superficies de fracturas en el microscopio electrónico, se trata de detectar la presencia de estas fases para verificar que se está evaluando la HAZ. En estos casos se analiza la microestructura del frente de fractura y se cuantifican las fases frágiles.

Los resultados de los ensayos Charpy $\mathrm{V}$ muestran que las probetas 2,3 y 4 presentan una importante dispersión, siendo la 3 la que presenta valores más elevados; en las probetas 5 y 6 , el efecto de revenido de las sucesivas pasadas es más efectivo debido a que son de menor espesor. El uso de precalentamiento en la probeta 6 presenta mejores resultados. En esta última probeta, tal como se expuso, las fases se presentan masivas.

A través de un estudio metalográfico del frente de fractura se comprueba si la fisura avanza a través
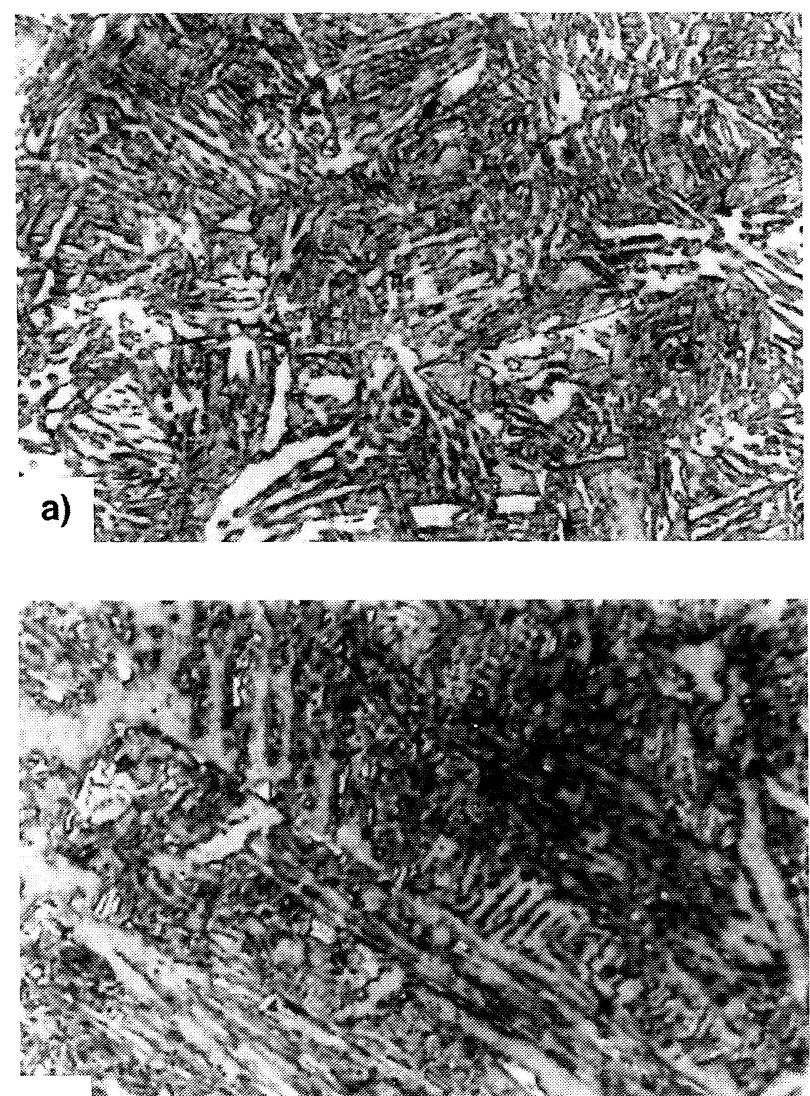

b)
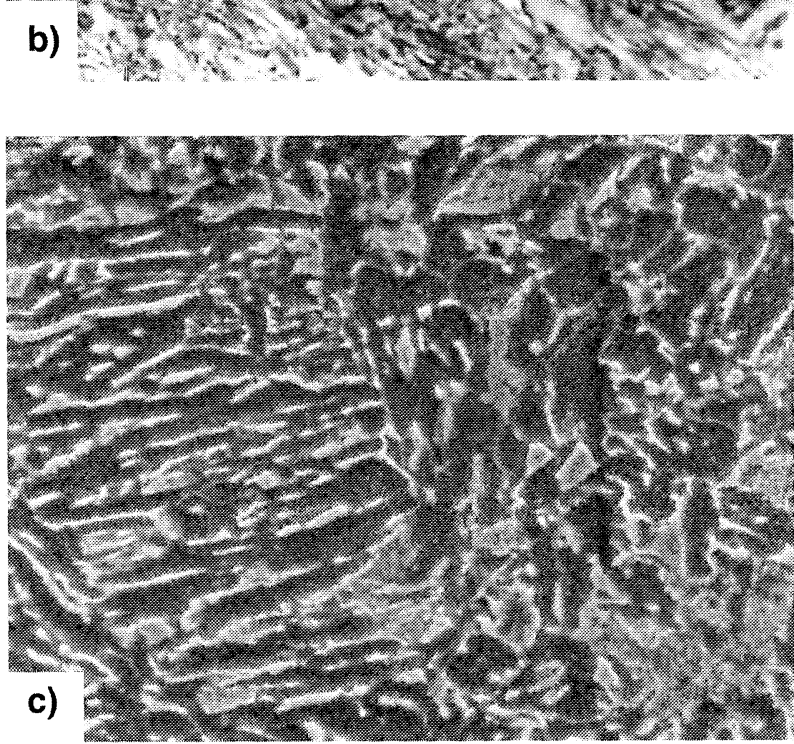

FIG. 3.- Observación microscópica: (a) Ataque Nital+Le Pera. $\times 400$. (b) Doble ataque electrolítico. $\times 400$. (c) $\mathrm{SEM} \times 3.020$.

(Reproducida en imprenta al 67\%).

FIG. 3.- Microscopic observation: (a) Nital + Le Pera. $\times 400$. (b) Two-stage electrolytic etching. $\times 400$ (c) $S E M \times 3,020$.

(Printed reproduction at $67 \%$ )

de la HAZ, en el metal base o en el de aporte. Los valores más bajos de energia se obtienen sobre la HAZ. En la figura 4 se cuantifica la energía frente al porcentaje de LBZ, donde se muestra cierta 


\section{TABLA III.- Ensayos Charpy V \\ TABLE III.- Charpy V tests}

\begin{tabular}{|c|c|c|c|c|c|c|}
\hline \multirow{2}{*}{$\begin{array}{l}\text { Material: } \\
\text { API 5LX60 }\end{array}$} & \multicolumn{6}{|c|}{ Charpy V, J } \\
\hline & Metal base & HAZ2 & HAZ3 & HAZ4 & HAZ5 & HAZ6 \\
\hline Tubos núms. 2, 3 y 4 & $132 *$ & 28 & 42 & $84 *$ & & \\
\hline Espesor = 10,3 mm & & 56 & 74 & 114\# & & \\
\hline Probeta $=10 \times 7,5 \times 55 \mathrm{~mm}$ & & 80 & $138 \#$ & 54 & & \\
\hline Temp. de ensayo $=-40{ }^{\circ} \mathrm{C}$ & & 96 & 86 & 50 & & \\
\hline & & 20 & $118 \#$ & 14 & & \\
\hline Tubos núms. 5 y 6 & $115^{*}$ & & & & 44 & 72 \\
\hline Espesor = 6,4 mm & & & & & 66 & 66 \\
\hline Probeta $=10 \times 5 \times 55 \mathrm{~mm}$ & & & & & 68 & 93 \\
\hline Temp. de ensayo $=-40^{\circ} \mathrm{C}$ & & & & & 46 & $95 \#$ \\
\hline & & & & & & 79 \\
\hline & & & & & & 82 \\
\hline & & & & & & 86 \\
\hline
\end{tabular}

* Valor promedio.

\# Metal base.

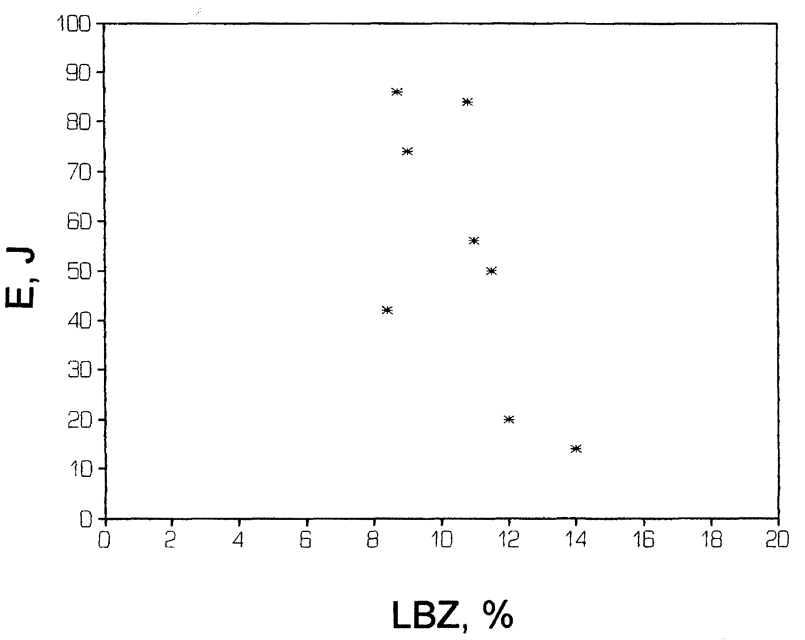

FIG. 4.- Energía en función del porcentaje de LBZ.

FIG. 4.-Energy vs. LBZ porcentage.

disparidad en los resultados, lo que indicaría que se debe evitar la presencia de estas fases. La observación del frente de fractura por SEM permite comprobar que la fisura avanza entre las fases y no a través de ellas. La figura 5 muestra una fase así como el resultado de la composicion química obtenido por medio de análisis con EDAX, donde se manifiesta un enriquecimiento de silicio.

Los barridos de dureza efectuados no permiten detectar valores elevados, ello se debe a la heterogeneidad de las microstructuras presentes, lo que dificulta que la impronta coincida con una fase frágil.

\section{CONCLUSIONES}

A pesar del bajo aporte térmico y de la importante cantidad de pasadas no puede evitarse la aparición de fases frágiles, estas se generan por un mecanismo complejo que involucra ciclos térmicos y efectos de composición.

Si bien podría esperarse que un precalentamiento favorezca la disolución de las mismas, este aumentaría el tiempo de enfriamiento $\mathrm{y}$, por lo tanto, la permanencia en el rango intercrítico de temperaturas.

El precalentamiento permite el control del tiempo de enfriamiento entre 800 y $500{ }^{\circ} \mathrm{C}$, pero debe tenerse en cuenta que:

- Un tiempo de enfriamiento largo origina fases frágiles (LBZ).

- Un tiempo de enfriamiento corto origina fases frágiles (martensita).

El hecho de partir de un material con microestructura en forma de bandas genera heterogeneidades que favorecen la presencia de las fases en distintas regiones, no se limita a la zona de grano grueso.

La identificación de las mismas es fácil a partir de métodos metalográficos, pero el análisis de su incidencia en las propiedades mecánicas es más complejo.

Para evaluar su incidencia en las propiedades mecánicas se recomienda el uso de ensayos de tenacidad a la fractura. En este caso, debido al pequeño 

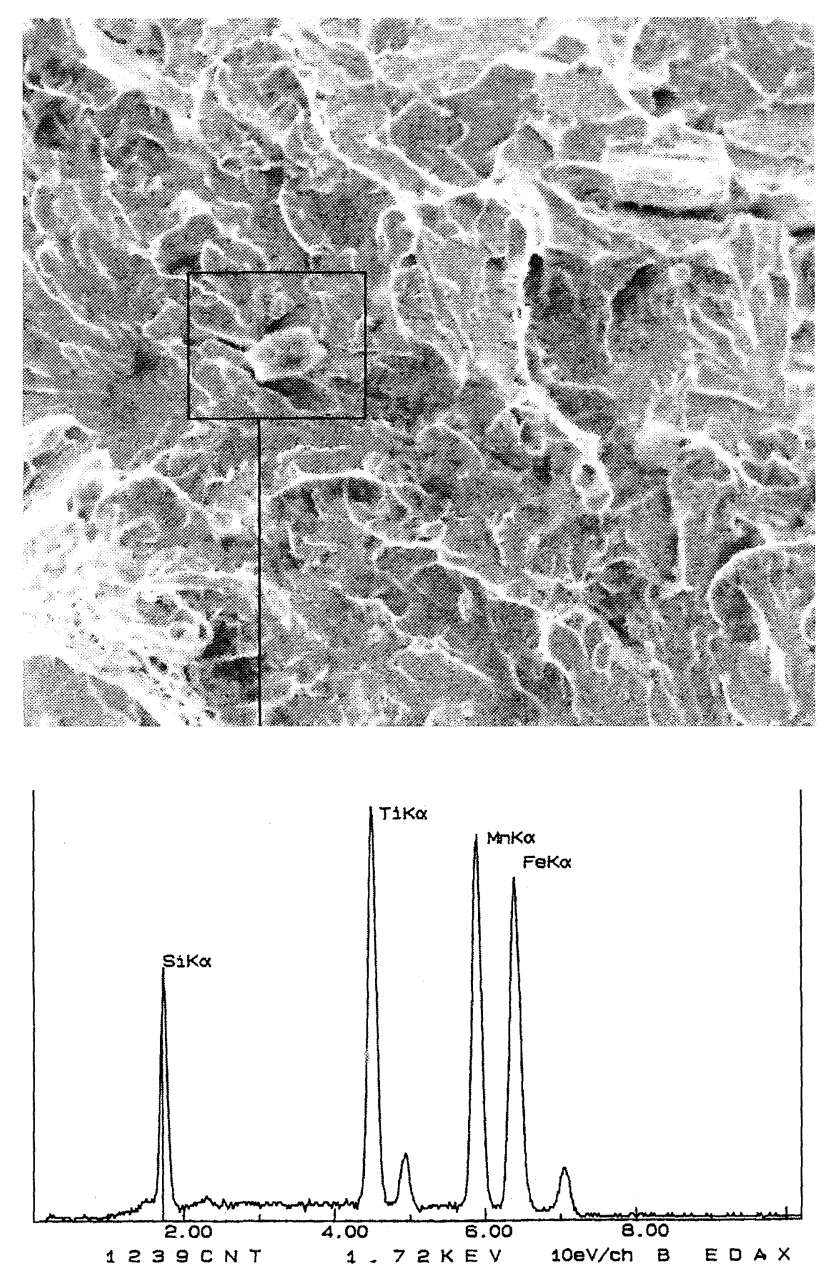

FIG. 5.- Superficie de fractura de la probeta Charpy V $\times 885$ Análisis con EDAX. (Reproducida en imprenta al $50 \%$ ).

FIG. 5.- Fracture surface from Charpy $V$ test. $\times 885$, EDAX analysis.

(Printed reproduction at $50 \%$ ).

espesor de las muestras, los valores obtenidos no fueron válidos por lo que no se incluyen los resultados.
Se puede ver, además, que los índices para evaluar la fisuración en frío no permiten considerar la presencia de fases frágiles.

\section{Agradecimientos}

Al Sector de Caracterización de Materiales del Centro Atómico Bariloche por el uso del SEM, y a Conarco-Electrodos S.A. por el suministro de los consumibles.

\section{REFERENCIAS}

(1) SHIGA, C. Proc. Int. Conf. on The metallurgy, welding, and qualification of microalloyed (HSLA) steel weldments. Microalloying Int.Inc. - AWS. Houston, Texas (EE.UU.) 1990: 327-350.

(2) Aihara, S. y Oкамото, K. Proc. Int. Conf. on The metallurgy, welding, and qualification of microalloyed (HSLA) steel weldments. Microalloying Int. Inc. - AWS. Houston, Texas (EE.UU.), 1990: 402- 426.

(3) HAZE T., Aihara S. IIW Doc. IX-1423-86.

(4) Doc. IIS-IIW-1128-91. Weld. World, 33 (1), 1994: 34-65.

(5) YurIOKA, N. Weld. World, 35 (6), 1995: 375-390.

(6) Matsuda, F., Fakuda, Y. et al. Weld. World, 37 (3) 1996: 134-154.

(7) Davies y KInG, J.E. Mat. Sci. Tech. A. 9, 1993: 8-15.

(8) Tillard, P. Verrier, T. Maurickx et al. Met. Trans. Vm. 26A, 1995: 447-457.

(9) CoE, F.R., BAILey, N. et al. Welding steels without hidrogen cracking. Abington Publ. ASM International. 46-72.

(10) British Standard 5135-1974.

(11) Yurioka, N. y KASUYA, T. Weld. World, 35 (5), 1995: 327-334.

(12) ANSI/AWS D1.1-94 Structural welding code. American Welding Society. 1994.

(13) SUZUKI, H. IIW Doc. IX-1704-93.

(14) Davies y King, J.E. Mater. Sci. Tech. A. 9, 1993: 8-15.

(15) Kim, B.C., LeE, S., Kim, N. y LeE, D. Met. Trans. V22 A, 1991: 131-149. 